\title{
MINIMIZING THE PRESENTATION OF A KNOT GROUP
}

\author{
MARK J. DUGOPOLSKI \\ Department of Mathematics \\ Southeastern Louisiana University \\ Hammond, Louisiana 70402 U.S.A. \\ (Received October 12, 1984)
}

ABSTRACT. A method for calculating the fundamental group of a knot is given. This method can be used to obtain group presentations with fewer generators and relators than other methods.

KEY WORDS AND PHRASES. Fundamental group, generator, relator. 1980 MATHEMATICS SUBJECT CLASSIFICATION CODE. $57 M 05$

1. INTRODUCTION.

In most recent books on knot theory, the fundamental group of a knot is determined by the Wirtinger method. For alternating knots of $n$ crossings, this method gives us a group with $n$ generators and $n$ relations. For non-alternating knots it is only slightly more economical. If one wants a simpler presentation, one then must make some algebraic observations and substitutions to reduce the presentation. In this paper, we describe a method for determining the fundamental group of a knot which can give us far fewer generators and relations than the Wirtinger method. The reduction in generators and relations is accomplished geometrically. This gives us a better understanding of the relationship between algebra and geometry in the fundamental group. This method is then used to show that all knots of seven or fewer crossings are one relator knots and all knots of, ight of nine crossings have presentations with two or fewer relaturs. All work here is in the piecewise linear category.

2. DETERMINING THE FUNDAMENTAL GROUP.

A knot is defined as a piecewise linear image of $S^{1}$ in $s^{3}$. We will think of the knot as being slightly thickened or as being a knotted torus. The fundamental group of a knot $K$ is $\pi_{1}\left(S^{3}-K\right)$, but we will often write $\pi_{1}(K)$. For our purposes we want to view the knot in the manner in which one would tie the knot with a rope having two ends. To accomplish this, we use the fact that $s^{3}$ is the union of two solid cubes identified on their boundaries. From $\mathrm{s}^{3}-\mathrm{K}$ we remove a cube which is pierced by the knot such that this section of the knot is unknotted. The knot then lies inside of a solid cube with its two ends on the boundary of the cube. Diagram 1 shows the trefoil in this way. 

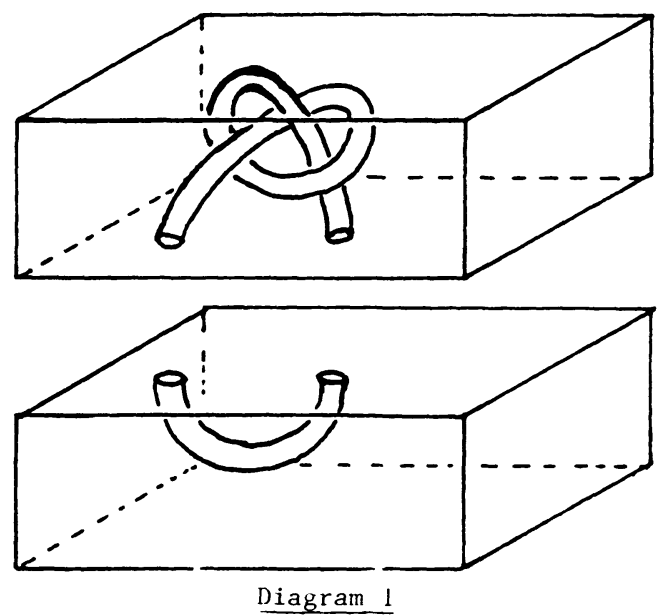

We now need to see that $\pi_{1}\left(S^{3}-K\right)$ is the same as the fundamental group of the solid cube with the knot drilled out of it. This is easily seen using Van Kampen's theorem as stated by Rolfsen [1]:

If $x=x_{1} \cup x_{2}$ and $x_{1} \cap x_{2}=x_{0}$, then to obtain $\pi_{1}(x)$ one throws together the generators and relations from $\pi_{1}\left(\mathrm{X}_{1}\right)$ and $\pi_{1}\left(\mathrm{X}_{2}\right)$ plus one relation for each generator of $\pi_{1}\left(X_{0}\right)$ which says that its image in $\pi_{1}\left(x_{1}\right)$ and $\pi_{1}\left(\mathrm{X}_{2}\right)$ are equal.

To determine $\pi_{1}(K)$ we start with a projection of $K$, just as in the Wirtinger method. We remove a cube at an undercrossing which lies adjacent to an overcrossing. We look at a projection of the knot onto the bottom of the cube, but keep the knot just slightly above the bottom plane, except at the ends which are attached to that plane. See Diagram 2 for an example using the knot $4_{1}$ of Rolfsen's table [1].

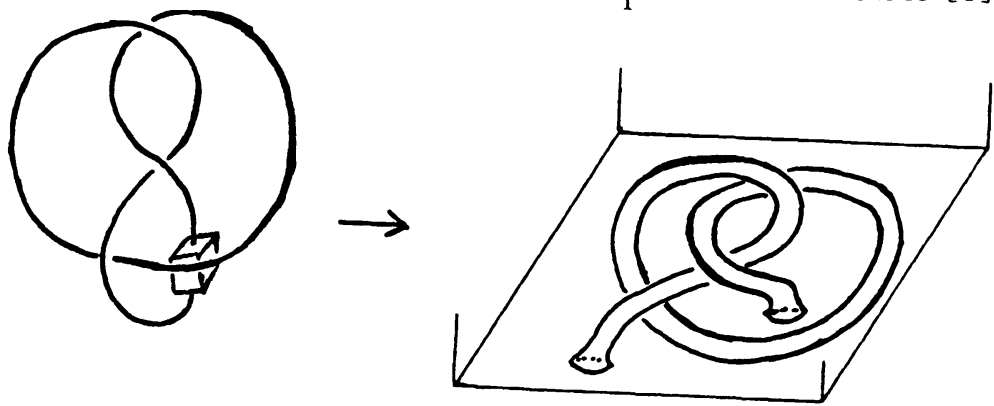

Diagram 2

Now at each overcrossing we drill out a hole coming up from the bottom plane and then back down as in Diagram 3. If we ignore the knot, $\pi_{1}$ of a cube with three un- 


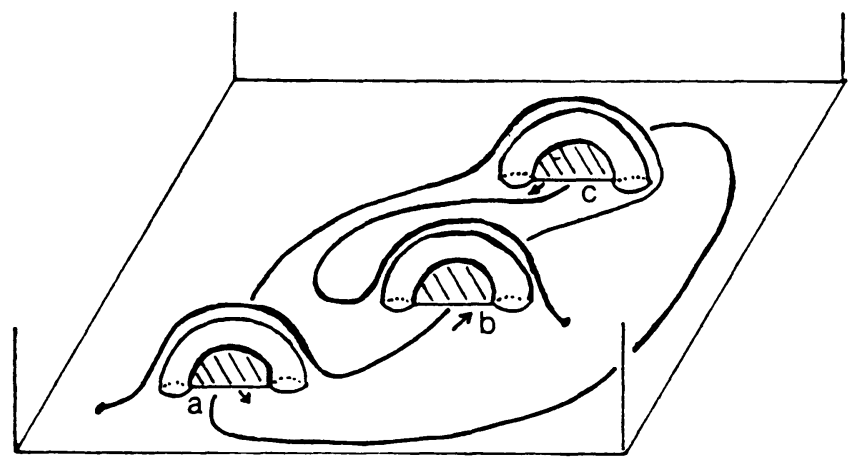

Diagram 3

knotted holes drilled out is free on three generators, $\{a, b, c\}$. The disks shown in Diagram 3 are thought of as the generators and the arrows shown by the disks are for orientation. A loop in this space determines a word in the free group according to which disks it passes through and in which direction, i.e., $b$ is with the arrow and $b^{-1}$ is against the arrow.

Now, since we separated the knot at an undercrossing which was adjacent to an overcrossing, there is a direction in which we can traverse this knot which starts with an overcrossing. Take the drilled out hole corresponding to this overcrossing and deform the cube with holes by pushing that first hole around the path of the knot, just as if we were tying the knot. See Diagram 4.

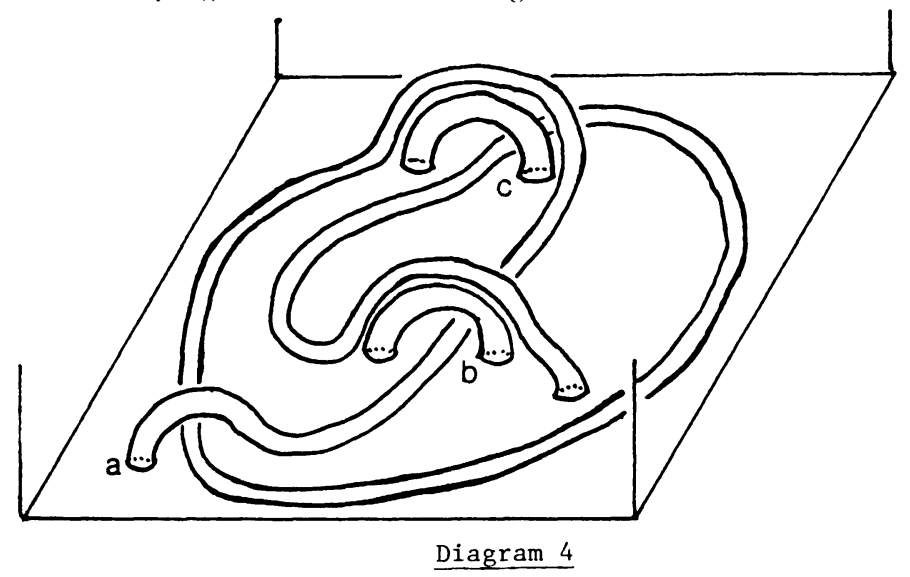

The fundamental group of the cube with holes is unchanged by this deformation, it is still the free group. We now fill in all of the holes except the one forming the knot. By Van Kampen's theorem, each hole we fill in introduces a relation into the free group. In our example we fill in $\mathrm{b}$ and $\mathrm{c}$, giving us a group with three generators and two relations as the fundamental group of the knot $4_{1}$. The Wirtinger method would have given four generators and four relations. Later, we will modify our method to see that $4_{1}$ is a one-relator knot.

3. DETERMINING THE RELATIONS.

To determine precisely what the relations are, we must keep track of how the disks under holes are deformed. We do this now for the knot $4_{1}$. When a pushes through 
b , b must be deformed so that a does not actually pierce b. See Diagram 5 . We keep stretching $b$ so that $b$ always stays out in front of $a$ as $a$ moves over the rest of the knot.

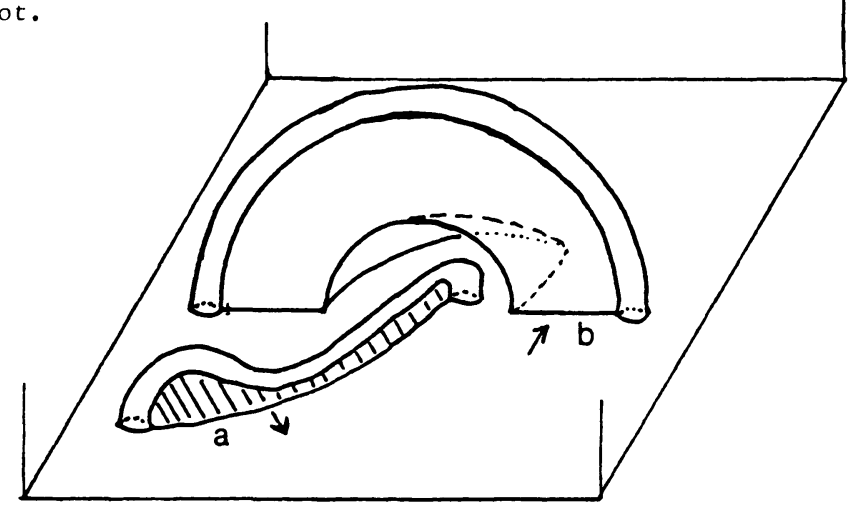

\section{Diagram 5}

To keep track of these disks, we draw a diagram of what is taking place on the bottom plane, using arrows to account for the orientations. See Diagram 6 . This diagram then tracks across $c$ as shown in Diagram 7. When we fill in $c$ we get the relation $b^{-1} a b c=1$ determined by the meridian shown in Diagram 7 . Since $b^{-1} a b=c^{-1}$ we can think of pushing $c^{-1}$ after this instead of $b^{-1} a b$. Now, from Diagram 4, we see that $c^{-1}$ goes under $a$, under $c$, and finally over $b$. We get the picture
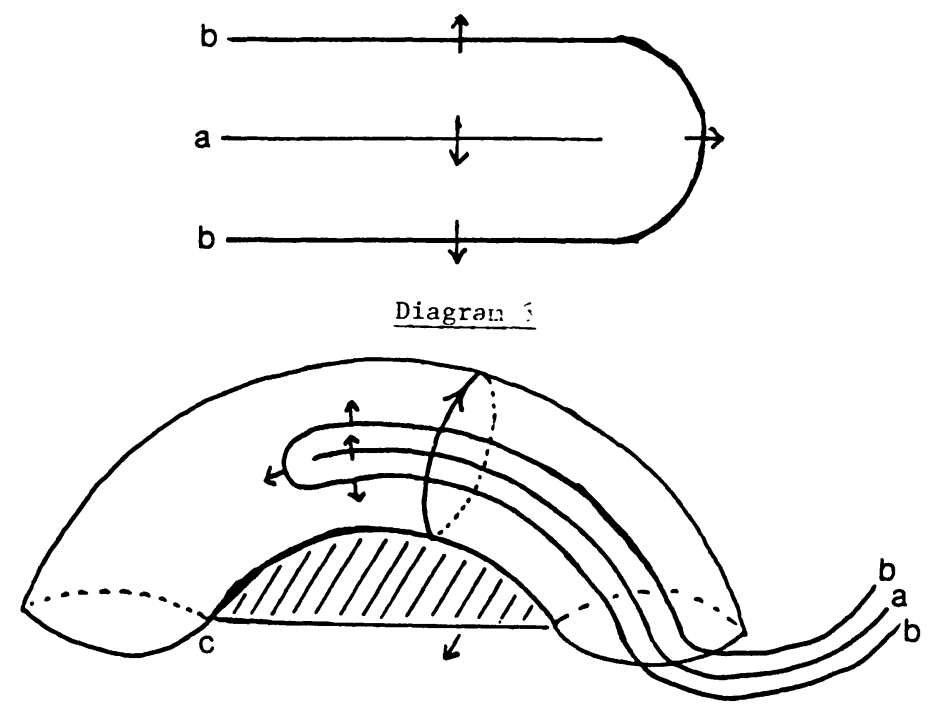

Diagram 7

shown in Diagram 8 and the relation $c^{-1} a^{-1} c^{-1} a c b=1$. Thus the fundamental group of the knot is $\left\{a, b, c ; b^{-1} a b=c^{-1}, c^{-1} a^{-1} c^{-1} a c=b^{-1}\right\}$. 


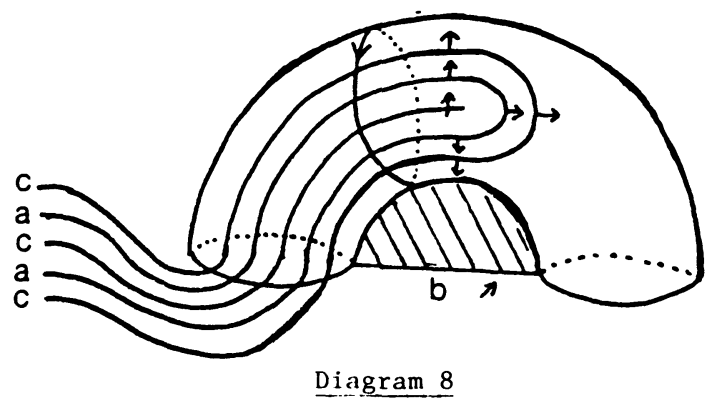

We can perform the process just described on any knot. If it is an alternating knot with $n$ crossings, we eliminate one crossing to get it inside the cube, drill holes at the remaining $n-l$ crossings, then fill in $n-2$ holes for relations. This proves the following theorem.

THEOREM 1. The fundamental group of every $n$ crossing alternating knot has a presentation with $\mathrm{n}-1$ generators and $\mathrm{n}-2$ relations.

The trefoil knot has a presentation with two generators and one relation and there is no presentation with fewer generators or relations for the trefoil. We conjecture that the trefoil is the only alternating knot for which the number of generators and relations guaranteed by Theorem 1 cannot be reduced.

A nonalternating knot has either two consecutive undercrossings or two consecutive overcrossings. We can assume there are two consecutive overcrossings. Then, in determining $\pi_{1}$, we drill our hole over more than one segment of the knot, reducing the number of generators and relations by one each. We may conclude the following theorem. THEOREM 2. The fundamental group of any nonalternating knot of $n$ crossings has a presentation with $n-2$ generators and $n-3$ relations.

4. MINIMIZING THE PRESENTATION.

For most knots we can find presentations with far fewer generators and relations than the numbers guaranteed by Theorems 1 and 2. As we described earlier, each hole drilled up from the bottom plane of the projection determines a relator and we need to drill holes until the fundamental group of the block is free.

We now look at the knot $4_{1}$ again, but this time we drill only one hole parallel to the knot as shown in Diagram 9.

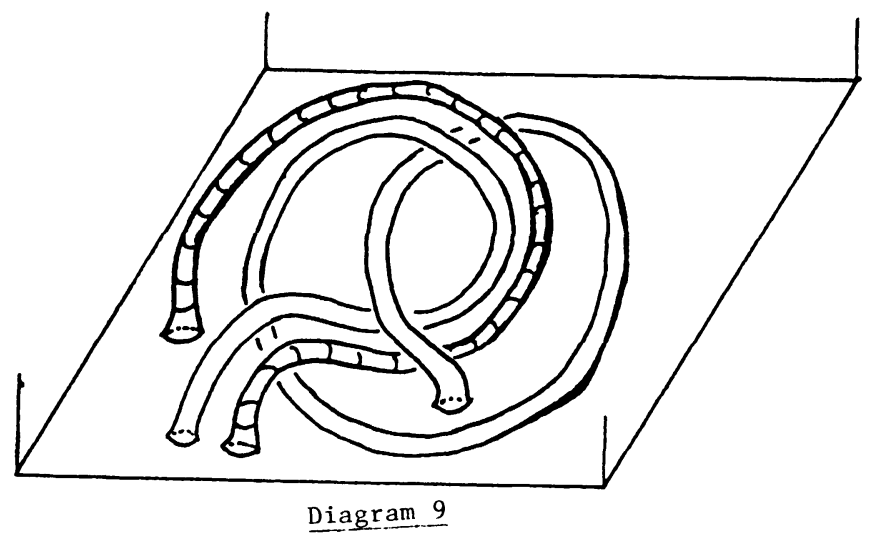


We then deform the block by sliding the knot over the hole to get the situation shown in Diagram 10.

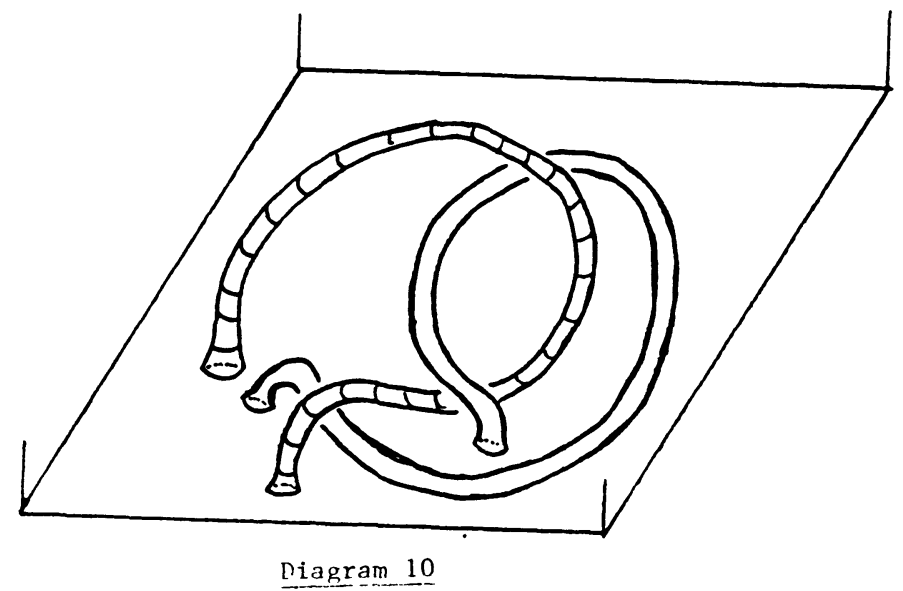

Further 1. formations as shown in Diagram 11 unravel the two holes and show us that the group is now free. Notice that to unravel the holes, we repeatedly slide one end of one hole out from under the other hole. Thus the knot $4_{1}$ is a one-relator knot. In order to sec what the relation is, we reverse the steps of Diagram 11 , keeping track of how the disks $a$ and $b$ are deformed in the process. We then slide $b$ over $a$ to get the relation $b^{-1} a b a^{-1} b^{-1} a b^{-1} a^{-1} b=a^{-1}$.

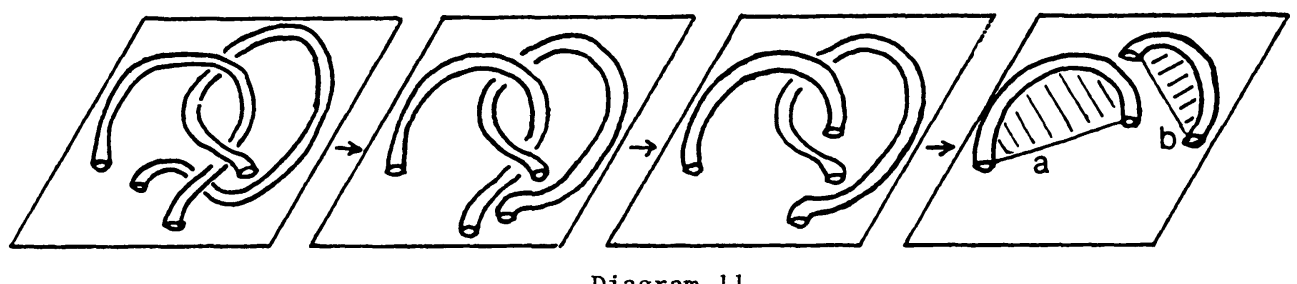

\section{Diagram 11}

Instead of drilling a hole alongside of the knot and then sliding the knot over the hole, we can accomplish the same thing by breaking the knot at an undercrossing and keeping the ends on the bottom plane of the cube. Thus, if we start with a knot projection and breaking it at two undercrossings causes the knot to unravel, then the knot is a one-relator knot. We must keep in mind that the unraveling is a deformation of the cube with holes and we can onlv slide an end of the knot out from under another segment of the knot. Diagram 12 shows that the knot $7_{6}$ is a one-relator knot.

The above method shows that in general a knot which unravels because of $n$ breaks at undercrossings has a presentation with $\mathrm{n}$ generators and $\mathrm{n}-1$ relations. It is a simple matter to go through a knot table to establish the following theorem. THEOREM 3. $\Lambda 11$ prime knots which have nrojections of seven or fewer crossings are one-relator knots. All prime knots which have projections with eight or nine crossings have presentations with two or fewer relations.

Although this unraveling method will establish a great many knots is ue-relator knots, the method does not appear 1 () be conclusive. Diagram 13 shows a one-relator 
knot where a is lid over b several times, but we annot break the projection in two places and uinrivel the knot.
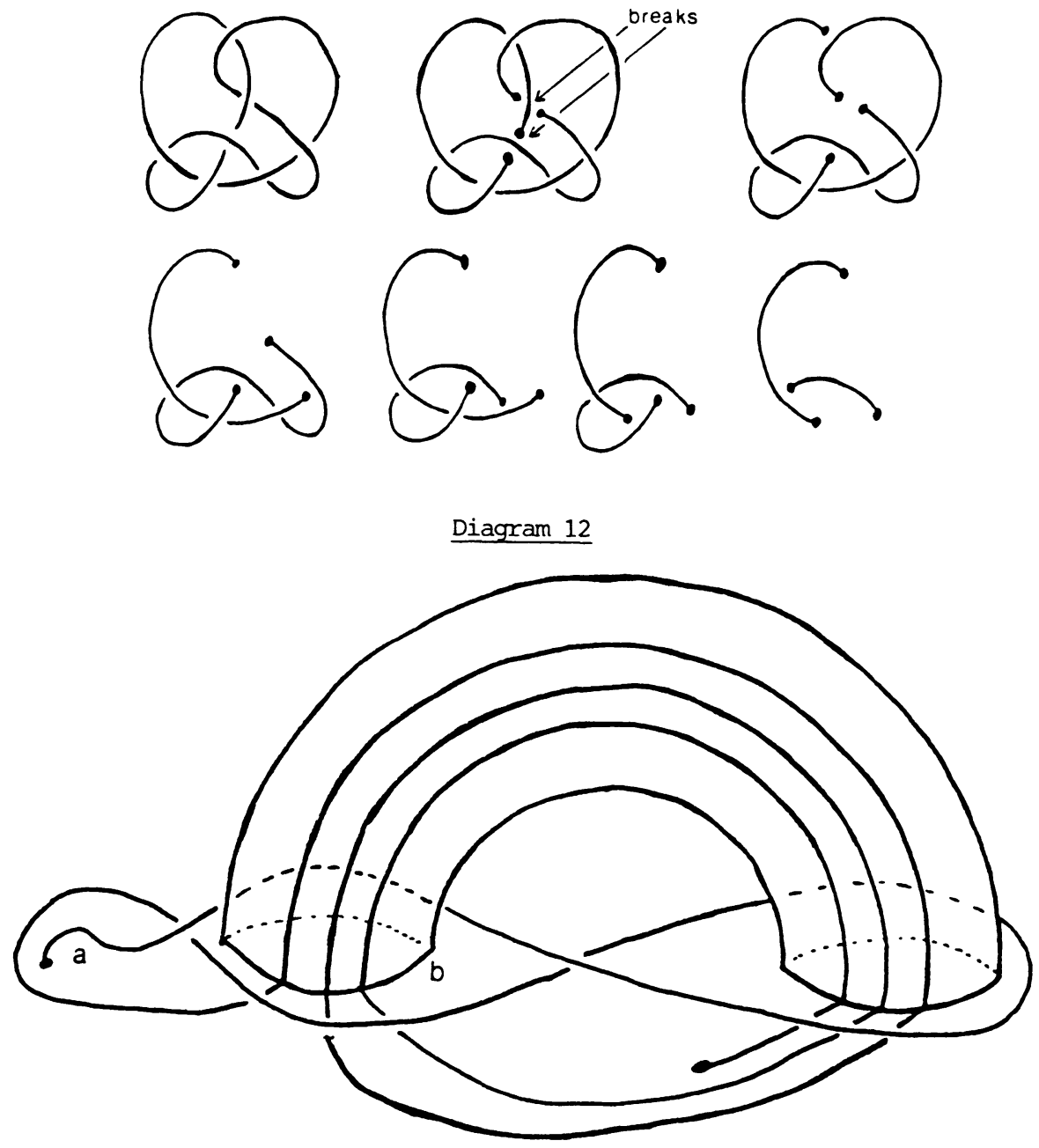

Diagram 13

Ideally, we would like to obtain an algorithm that would determine where to drill the holes and the minimum number of holes required in order to make the group free. This seems to be a difficult problem. Diagram 14 shows how to construct a knot whose group can be presented with two relators, but where the two drilled holes would be difficult to locate. This example shows that the holes used for the relators can be knotted. Holes a, b, and $c$ are drilled in the cube, $b$ is slid around $c$ in order to knot $b$, $a$ is slid over $b$ several times, then $b$ and $c$ are filled in.

In spite of the difficulties that may arise in finding where to drill the holes, we still believe there is a close relatiunship between the drilled holes and the relations of the knot group. We conjecture that $\pi_{1}$ of any one-relator knot can be made free by a single hole drilled in the solid cube containing the knot. We also conjecture that if $\pi_{1}$ of a knot is known to have a presentation with $n$ relations then 
the knot can be formed by drilling $n+1$ holes in a cube, sliding these holes around and filling in $n$ of the holes.
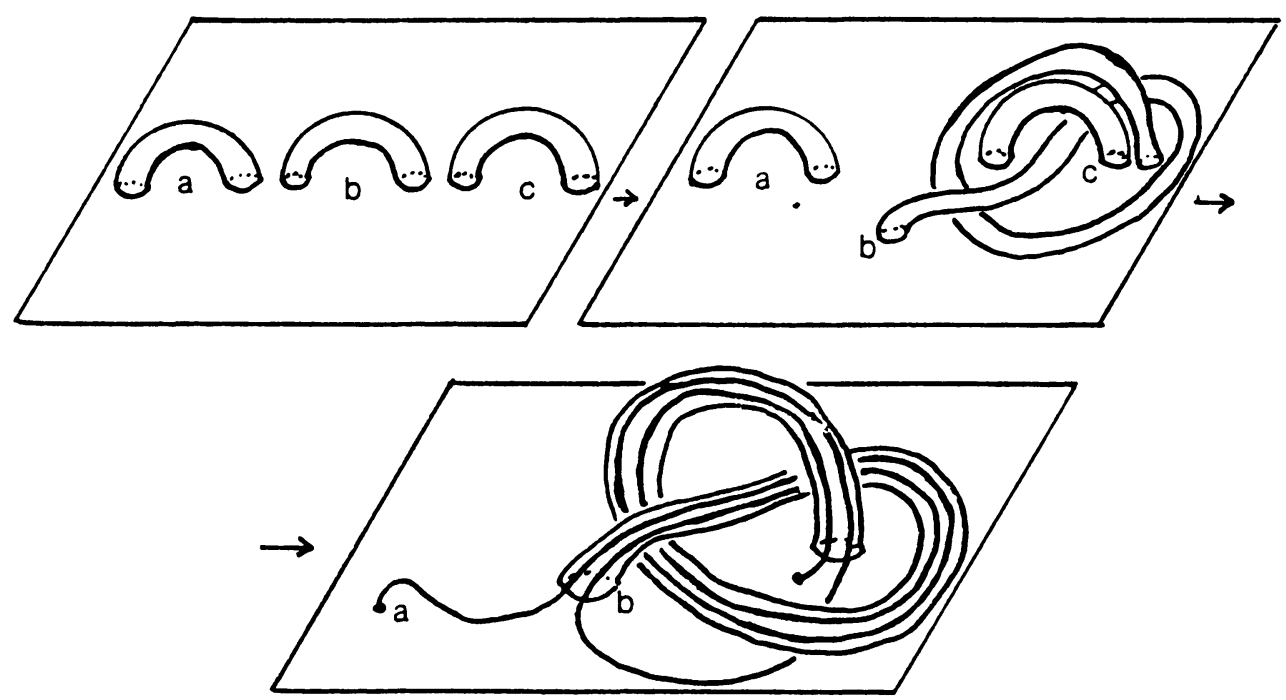

Diagram 14

REFERENCES

1. ROLFSEN, D. Knots and Links, Berkeley, CA, Publish Or Perish, Inc. 1976. 


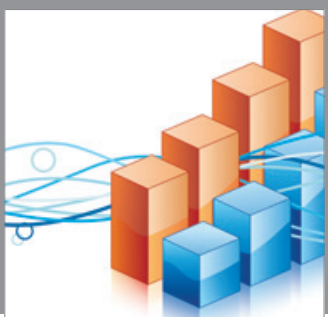

Advances in

Operations Research

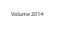

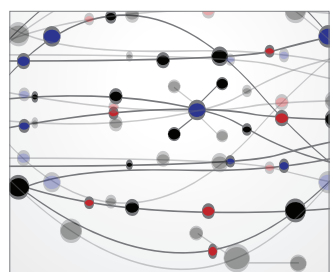

\section{The Scientific} World Journal
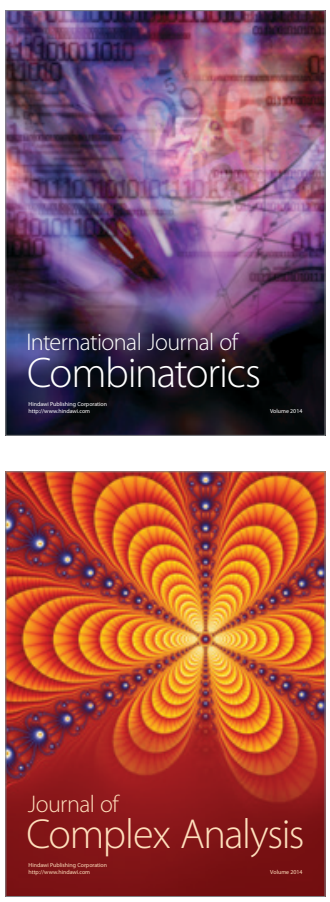

International Journal of

Mathematics and

Mathematical

Sciences
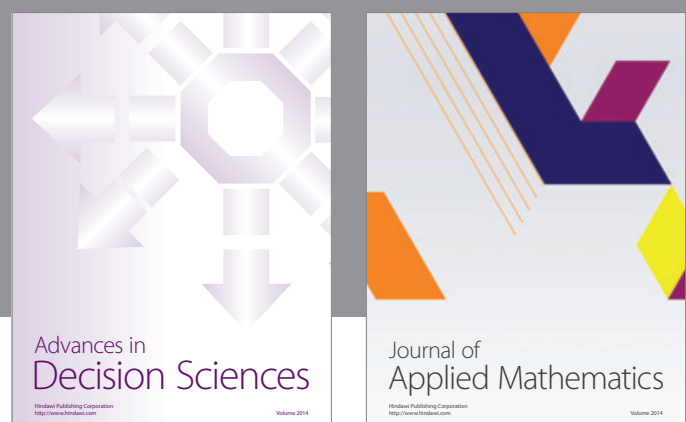

Journal of

Applied Mathematics
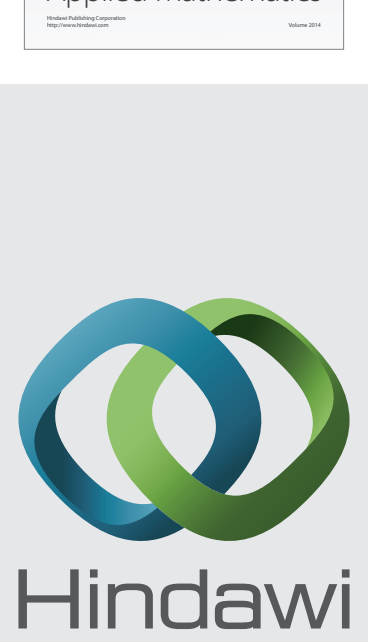

Submit your manuscripts at http://www.hindawi.com
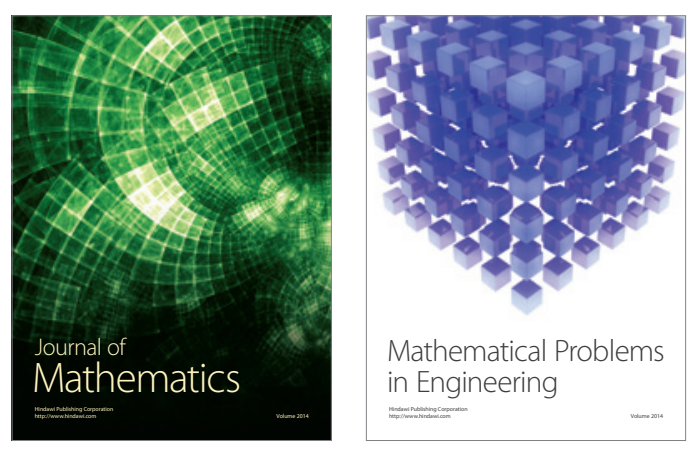

Mathematical Problems in Engineering
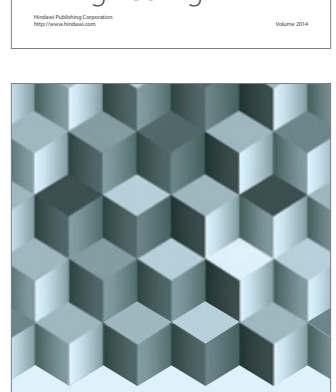

Journal of

Function Spaces
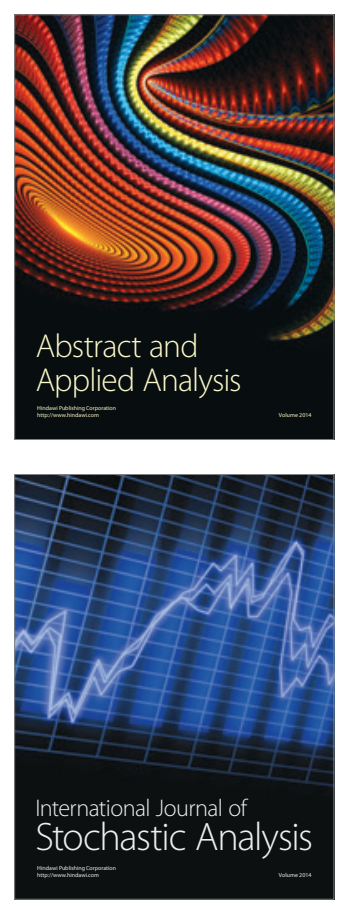

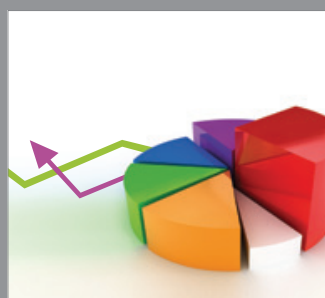

ournal of

Probability and Statistics

Promensencen
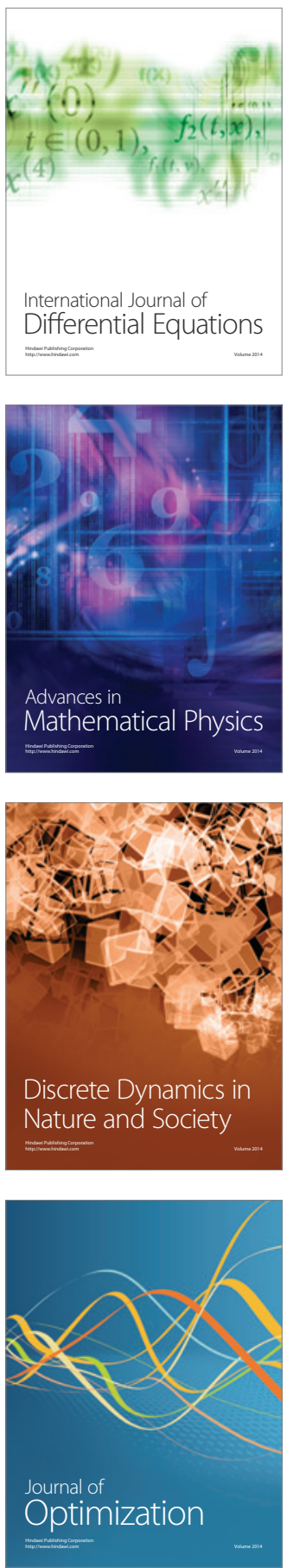